\title{
Comparación entre líneas de ratones en el ensayo de aberraciones cromosómicas en médula ósea
}

\section{Comparison between mice lines in bone marrow chromosomal aberrations testing}

\author{
Daniel Arencibia A, ${ }^{1 *}$ Ms.C, Luis Rosario F, ${ }^{2}$ Ms.C, Alexis Vidal N, ${ }^{3}$ Ph.D.
}

\begin{abstract}
${ }^{1}$ Instituto Finlay, Vicepresidencia de Investigaciones, Habana, Cuba. ${ }^{2}$ Instituto de Farmacia y Alimentos (IFAL, U.H), Habana, Cuba. ${ }^{3}$ Facultad de Biología (U.H), Habana, Cuba. *Correspondencia: darencibia@finlay.edu.cu
\end{abstract}

Recibido: Agosto de 2011; Aceptado: Febrero de 2012.

\section{RESUMEN}

Objetivo. El objetivo de este trabajo fue comparar los índices espontáneos e inducidos en ratones de ambos sexos de las líneas Balb/c, NMRI, OF-1 y C57BL/6/cenp, mediante el ensayo de aberraciones cromosómicas en células de la médula ósea. Materiales y métodos. Con este fin se determinó la línea más eficiente, sobre la base de la aparición significativa de índices espontáneos más bajos e índices inducidos altos a sustancias mutagénicas como la ciclofosfamida (CF). Resultados. Se obtuvo como resultado que la línea Balb/c en ambos sexos difiere significativamente de las demás líneas evaluadas. En esta línea se encontró el menor número de células totales con aberraciones estructurales y un mayor número de inducción de aberraciones cromosómicas cuando es utilizada la CF como mutágeno. Conclusiones. Este trabajo permitirá utilizar la mejor línea de ratones como biomodelo en este ensayo de genotoxicidad, además que le confiere a esta técnica citogenética mayor veracidad y robustez.

Palabras clave: Aberraciones cromosómicas, médula ósea, ratón (Fuente: AIMS).

\begin{abstract}
Objective. The aim of this study was to compare spontaneous and induced rates in mice of both sexes of lines BALB / c, NMRI, OF-1 and C57BL/6/ cenp, by testing chromosomal aberrations in bone marrow cells. Materials and methods. For this purpose, the most efficient line was determined, based on the significant appearance of lower spontaneous rates and higher induced rates to mutagen substances such as cyclophosphamide (CP). Results. The result obtained was that line Balb / c, in both sexes, differs significantly from the other lines tested. The lesser total number of cells with structural aberrations was found in this line and a greater number of induction of chromosomal aberrations when CP was used as a mutagen. Conclusions. This work will give way to the use of the best line of mice as bio model in this genotoxicity test, and will also give this cytogenetic technique greater veracity and robustness.
\end{abstract}

Key words: Bone marrow, Chromosomal aberrations, mice (Source: AIMS). 


\section{INTRODUCCIÓN}

Por lo general los estudios mutagénicos y genotóxicos en la toxicología experimental, se realizan cuando ya se han agotado todos los estudios de toxicidad aguda potencial del producto a evaluar en dos especies y por al menos dos vías (1). Dentro de esta batería de ensayos se encuentra el de aberraciones cromosómicas. Esta técnica citogenética in vivo es a corto plazo, de gran sensibilidad y útil para detectar fundamentalmente aberraciones cromosómicas estructurales.

Con los mutágenos químicos, la mayor parte de las aberraciones inducidas son de tipo cromatídico. En este método, se utilizan células de médula ósea de mamíferos expuestos, por vías apropiadas, a las sustancias de ensayo y sacrificados a intervalos sucesivos (2). El tejido diana es la médula ósea por estar más vascularizado y por contener una población de células de ciclo muy corto las cuales pueden aislarse y tratarse con facilidad (2).

Como controles positivo se utilizan sustancias mutagénicamente activas, con mecanismos de mutación conocida, dentro de estas la más difundida ha sido la ciclofosfamida (CF), caracterizada como un clastógeno químico potente $(1,2)$. Las sustancias utilizadas como controles positivos en los estudios de genotoxicidad in vivo se pueden administrar por vía y dosis diferentes al producto farmacéutico en investigación (2).

Por otro lado se plantea que durante las investigaciones de un mismo producto a evaluar mediante el tamizaje genotoxicológico se utilizan diferentes líneas sin tener en cuenta, la más eficiente para detectar daño en el ADN. Además de que las líneas de ratones producidas en Cuba hasta el momento no habían sido evaluadas $y / 0$ exploradas teniendo en cuenta su eficiencia genética para detectar sustancias con baja, media o alta genotoxicidad. Además el estudio profundo de nuestros biomodelos permitirá utilizar el mínimo de animales y obtener resultados fiables; por lo que disminuirán las repeticiones de estos estudios tan costosos y engorrosos.

Para la evaluación de nuevos productos es necesario conocer la frecuencia espontánea e inducida de aparición de cada uno de los fenómenos que estudia la toxicología genética en el biomodelo a utilizar. Esto permitiría conocer nuevas sustancias con potencial genotóxico, así como la medida en que se manifiesta. Por otro lado al ser estas pruebas tan decisivas en la evolución positiva o negativa de un nuevo producto es necesario buscar el biomodelo animal ideal (3).

El objetivo de este trabajo fue comparar los índices espontáneos e inducidos en ratones de ambos sexos de las líneas Balb/c, NMRI, OF-1 y C57BL/6/cenp, mediante el ensayo de aberraciones cromosómicas en células de la médula ósea para determinar la línea más eficiente, sobre la base de la aparición significativa de índices espontáneos más bajos e índices inducidos altos a sustancias mutagénicas como la CF.

\section{MATERIALES Y MÉTODOS}

Animales. Se utilizaron ratones de ambos sexos adultos jóvenes (6-7 semanas), de las líneas Balb/c, NMRI, OF-1 y C57BL/6/cenp. El peso corporal de los animales osciló entre 25 y $30 \mathrm{~g}$ al término de la cuarentena, donde se mantuvieron en condiciones controladas: temperatura $\left(25 \pm 2^{\circ} \mathrm{C}\right)$, humedad relativa $(60 \% \pm 10 \%)$ y ciclos de luz-oscuridad de $12 \mathrm{~h}$. El acceso al agua y al alimento (pienso estándar para esta especie suministrado por el CENPALAB), fue ad libitum. Estas características fueron comunes para todos los grupos experimentales evaluados en este ensayo. Toda la manipulación de los animales se realizó de acuerdo con los principios éticos internacionales para el uso de los animales de Laboratorios CCAC (4).

Procedimientos éticos. Los autores declaramos que este trabajo fue confeccionado sobre la base de buenas prácticas de laboratorio preclínico presentes en el reglamento nacional de aprobación de protocolos de investigación de la República de Cuba. Además se declara por parte de los autores del artículo que al comenzar esta investigación se obtuvo por escrito el consentimiento de aprobación del protocolo e informe por el comité institucional de ética para la experimentación animal de nuestra institución.

Grupos experimentales. En todos los grupos experimentales, la sustancia se administró en el horario de 10:30-11:30 a.m, y las concentraciones a administrar de cada sustancia se ajustaron semanalmente en función del aumento del peso corporal. Los animales se distribuyeron aleatoriamente (10 ratones/ grupo/sexo).

En el grupo experimental 1 se utilizaron animales no tratados, a los cuales se les realizó la técnica de entubación gástrica durante 14 días. 
En el grupo experimental 2 se utilizó Tween $®$ 65 al 2\%, vehículo utilizado en la mayoría de las preparaciones de sustancias oleosas, útil como agente tensoactivo (5). Este producto fue adquirido de la firma Applichem con CMC $\left(25^{\circ} \mathrm{C}\right)=5.9 \times 105 \mathrm{~mol} / \mathrm{L}$ y solubilidad $\left(25^{\circ} \mathrm{C}\right) \mathrm{de}$ $100 \mathrm{~g} / \mathrm{L}$ en $\mathrm{H} 2 \mathrm{O}$ y número de lote $9 \mathrm{~B} 006892$. En el grupo experimental 3 se utilizó $\mathrm{NaCl}$ al $0.9 \%$ como segundo grupo solvente, ya que está demostrado que el mismo es el disolvente de la mayoría de las sustancias hidrofílicas a preparar (6). Este solvente fue adquirido de la firma $\mathrm{BDH}$ (BDC) con un $99 \%$ de pureza y número de lote L451491932. Ambos productos fueron preparados 2 horas antes de la administración; se administraron por vía oral durante 14 días.

En el grupo experimental 4 se utilizó como control positivo la $\mathrm{CF}, \mathrm{N}, \mathrm{N}$-bis (cloruro de etilo)-N', O-esterdiamida del ácido fosfórico propinel(C17H15Cl2N2O5P), en dosis de $50 \mathrm{mg} / \mathrm{kg}$, por vía intraperitoneal. Este mutágeno fue adquirido por la firma comercial mexicana Lemery SA bajo la marca LEDOXINA $®$, con un $98 \%$ de pureza y lote de fabricación número 27555/2009. El mismo se diluyó en disolución salina $(\mathrm{NaCl})$ al $0.9 \%$. La disolución se administró inmediatamente después de ser preparada, 48 horas y luego a las 24 horas antes de la eutanasia $(7,8)$.

Observaciones clínicas. Se realizaron dos observaciones clínicas diarias, en el horario comprendido entre las 8:30-10:30 a.m. y en el horario de la tarde 3:00-4:30 p.m. Durante cada observación se tuvo en cuenta el estado clínico general del animal, lo cual incluyó la palpación para la detección de lesiones, posibles afectaciones respiratorias, del sistema nervioso, cardiovascular, gastrointestinal, estado de la piel, pelo, coloración de las mucosas y ojos.

Ensayo de aberraciones cromosómicas en células de la médula ósea. En el horario de la mañana (4 horas antes del sacrificio), la división celular en metafase se detuvo utilizando colchicina (4 mg/kg, vía i.p). Un fémur de cada animal fue extraído y la cavidad medular se lavó con $3 \mathrm{~mL}$ de suero bovino fetal (SBF). La suspensión celular se centrifugó, eliminándose el sobrenadante. Después de un tratamiento hipotónico de las células del botón con $\mathrm{KCL}(0.075 \mathrm{M})$, se realizó una segunda centrifugación. El botón celular se fijó en una mezcla de metanol-ácido acético glacial (3:1) durante $15 \mathrm{~min}$. Se realizaron 3 fijaciones con centrifugaciones sucesivas, y se extendieron en láminas húmedas con enfriamiento previo. Las láminas se secaron al aire y se tiñeron con solución de Giemsa al 10\% durante 30-35 min
$(2,7)$. Se contabilizaron 100 metafases por animal, determinándose el número de células con aberraciones (rupturas e intercambios cromosómicos y cromatídicos) y frecuencia de gaps. También se calculó el índice mitótico IM\% (porcentaje de metafases en 1000 células leíbles) y el número de células con poliploidía en 1000 células leíbles, todas las determinaciones fueron leídas por dos observadores, para luego establecer un promedio entre ambas $(2,7)$.

Eutanasia. A todos los animales se les practicó la eutanasia por dislocación cervical teniendo en cuenta el tiempo de exposición a cada una de las sustancias evaluadas (6).

Análisis estadístico. Se procedió a verificar los supuestos para realizar el análisis de varianza en la variable continua índice mitótico, los resultados obtenidos están distribuidos normalmente (normalidad, según el test de Kolmogorov-Smirnov), existiendo dependencia entre las observaciones y presentan homogeneidad de varianzas (test de levene). Por lo cual se analizó con el uso de esta prueba, siendo el nivel de significación establecido de $\alpha=0.05(2,7)$. Las variables categóricas, número de células con aberraciones, frecuencia de gaps y el número de células con poliploidía, se analizaron mediante la prueba de $\chi^{2}$, el nivel de significación establecido fue de $\alpha=0.01(2,7)$. Todos los análisis se realizaron empleando el Statsoft for Windows. StatSoft, Inc. (2003). STATISTICA (data analysis software system), versión 6.

\section{RESULTADOS}

No se evidenció síntomas clínicos indicativos de toxicidad. Esto reafirma que la dosis utilizada no fue altamente tóxica a nivel sistémico, pero se logró un efecto importante en las células dianas analizadas.

Como resultado general se detectaron dentro de la variable número de células con aberraciones en mayor frecuencia las aberraciones de tipo cromatídicas, tal y como está descrito en la literatura. Este tipo de aberraciones son en su mayoría las que esta técnica citogenética es capaz detectar (estructurales).

Teniendo en cuenta el número total de células con aberraciones en la línea Balb/c se encuentra de forma espontánea entre 7-10 en los machos y entre 8-9 en las hembras (Tabla 1 y 2 ). Sin embargo la CF es capaz de inducir en esta línea hasta 175 células con aberraciones en los machos y 192 en las hembras. Para el caso de la 
Tabla 1. Comparación entre ratones Balb/c y OF-1 de ambos sexos en el ensayo de aberraciones cromosómicas en células de la médula ósea.

\begin{tabular}{|c|c|c|c|c|}
\hline $\begin{array}{c}\text { Grupos } \\
\text { Experimentales }\end{array}$ & $\operatorname{IM}(\%)^{a}$ & CP & Gaps $^{\text {b }}$ & \#C \\
\hline \multicolumn{5}{|c|}{ Ratones Balb/c de ambos sexos } \\
\hline \multicolumn{5}{|c|}{ Machos } \\
\hline Control Negativo & $\begin{array}{c}5.65 \pm \\
0.56 a\end{array}$ & 1 & $4 a$ & $7 a$ \\
\hline Sustancia Vehículo 1 & $\begin{array}{c}5.49 \pm \\
0.53 a\end{array}$ & 0 & $6 a$ & $8 a$ \\
\hline Sustancia Vehículo 2 & $\begin{array}{c}5.86 \pm \\
0.20 \mathrm{a}\end{array}$ & $0 a$ & $7 a$ & $10 a$ \\
\hline Control Positivo (CF) ${ }^{1}$ & $\begin{array}{l}3.89 \pm \\
0.24 * a\end{array}$ & $14 * * a$ & $47 * * a$ & $175^{* * a}$ \\
\hline \multicolumn{5}{|c|}{ Hembras } \\
\hline Control Negativo & $\begin{array}{c}5.98 \pm \\
0.22 a\end{array}$ & $1 a$ & $6 a$ & $8 a$ \\
\hline Sustancia Vehículo 1 & $\begin{array}{l}4.98 \pm \\
0.79 a\end{array}$ & 0 & $5 a$ & $8 a$ \\
\hline Sustancia Vehículo 2 & $\begin{array}{c}5.12 \pm \\
0.63 a\end{array}$ & 1 & $2 a$ & $9 a$ \\
\hline Control Positivo (CF) ${ }^{1}$ & $\begin{array}{l}3.93 \pm \\
0.84 * a\end{array}$ & $18 * * a$ & $44 * * a$ & $192 * * a$ \\
\hline \multicolumn{5}{|c|}{ Ratones OF-1 de ambos sexos } \\
\hline \multicolumn{5}{|c|}{ Machos } \\
\hline Control Negativo & $\begin{array}{c}5.42 \pm \\
0.31\end{array}$ & 2 & 7 & 13 \\
\hline Sustancia Vehículo 1 & $\begin{array}{c}5.20 \pm \\
0.56\end{array}$ & 1 & 8 & 15 \\
\hline Sustancia Vehículo 2 & $\begin{array}{c}5.71 \pm \\
0.20\end{array}$ & 1 & 5 & 14 \\
\hline Control Positivo (CF) ${ }^{1}$ & $\begin{array}{c}3.27 \pm \\
0.40 *\end{array}$ & $19 * *$ & $55 * *$ & $239 * *$ \\
\hline \multicolumn{5}{|c|}{ Hembras } \\
\hline Control Negativo & $\begin{array}{c}5.66 \pm \\
0.42\end{array}$ & 0 & 9 & 16 \\
\hline Sustancia Vehículo 1 & $\begin{array}{c}5.46 \pm \\
0.30\end{array}$ & 1 & 8 & 14 \\
\hline Sustancia Vehículo 2 & $\begin{array}{c}4.99 \pm \\
0.18\end{array}$ & 1 & 6 & 12 \\
\hline Control Positivo (CF) ${ }^{1}$ & $\begin{array}{l}3.38 \pm \\
0.35^{*}\end{array}$ & $22 * *$ & $58 * *$ & $253 * *$ \\
\hline
\end{tabular}

$\mathrm{CP}=$ Células con Poliploidía ${ }^{\mathrm{b}}$ \#C=\# de Células con aberraciones ${ }^{\mathrm{b}}$; CF (Ciclofosfamida); ${ }^{1}$ Administración por vía intraperitoneal. aX \pm D.E. de un total de 10000 células/grupo/ serie para un total de 20000 células evaluadas; $* p<0.05$; ANOVA (Comparación contra el control negativo en cada línea evaluada y sexo); $b{ }^{* *} p<0.01$; prueba no paramétrica $\mathrm{X}^{2}$ (Comparación contra el control negativo en cada línea evaluada y sexo); $a=p<0.05$ (Difiere al comparar entre líneas teniendo en cuenta la misma variable. grupo experimental. prueba estadística y sexo.

línea NMRI este resultado de forma espontánea se encuentra en el rango de 7-11 células totales y en las hembras es algo superior y significativo al compararlos con la Balb/c siendo de 10-14 células totales con aberraciones. Sin embargo la línea de ratones NMRI demostró ser más susceptible a la CF que la Balb/c; ya que este mutágeno logró inducir hasta 223 células
Tabla 2. Comparación entre ratones NMRI y C57BL/6/ cenp de ambos sexos en el ensayo de aberraciones cromosómicas en células de la médula ósea.

Grupos
Experimentales IM(\%) $^{\text {a }} \quad$ CP $\quad$ Gaps $^{b} \quad$ \#C

Ratones NMRI de ambos sexos

\begin{tabular}{|c|c|c|c|c|}
\hline \multicolumn{5}{|c|}{ Machos } \\
\hline Control Negativo & $\begin{array}{l}5.42 \pm \\
0.43\end{array}$ & 1 & 4 & 9 \\
\hline Sustancia Vehículo 1 & $\begin{array}{c}5.33 \pm \\
0.50\end{array}$ & 0 & 6 & 7 \\
\hline Sustancia Vehículo 2 & $\begin{array}{c}5.10 \pm \\
0.32\end{array}$ & 0 & 3 & 11 \\
\hline Control Positivo (CF) ${ }^{1}$ & $\begin{array}{c}3.52 \pm \\
0.21^{*}\end{array}$ & $15^{* *}$ & $45^{* *}$ & $223 * *$ \\
\hline \multicolumn{5}{|c|}{ Hembras } \\
\hline Control Negativo & $\begin{array}{c}5.12 \pm \\
0.23\end{array}$ & 1 & 8 & 12 \\
\hline Sustancia Vehículo 1 & $\begin{array}{c}5.21 \pm \\
0.55\end{array}$ & 2 & 2 & 14 \\
\hline Sustancia Vehículo 2 & $\begin{array}{c}5.03 \pm \\
0.48\end{array}$ & 1 & 5 & 10 \\
\hline Control Positivo (CF) ${ }^{1}$ & $\begin{array}{c}3.68 \\
\pm 0.12 *\end{array}$ & $20 * *$ & $51 * *$ & $219 * *$ \\
\hline
\end{tabular}

Ratones C57BL/6/cenp de ambos sexos

\begin{tabular}{|c|c|c|c|c|}
\hline \multicolumn{5}{|c|}{ Machos } \\
\hline Control Negativo & $\begin{array}{l}5.82 \pm \\
0.95\end{array}$ & 2 & $10 a$ & $17 a$ \\
\hline Sustancia Vehículo 1 & $\begin{array}{c}5.20 \pm \\
0.28\end{array}$ & 0 & 9 & $16 a$ \\
\hline Sustancia Vehículo 2 & $\begin{array}{c}5.59 \pm \\
0.33 a\end{array}$ & $3 a$ & $11 a$ & $16 a$ \\
\hline Control Positivo (CF) ${ }^{1}$ & $\begin{array}{c}3.46 \pm \\
0.29 *\end{array}$ & $21 * * a$ & $60 * * a$ & $254 * * a$ \\
\hline \multicolumn{5}{|c|}{ Hembras } \\
\hline Control Negativo & $\begin{array}{c}5.21 \pm \\
0.71\end{array}$ & 3 & 8 & $18 a$ \\
\hline Sustancia Vehículo 1 & $\begin{array}{l}4.96 \pm \\
0.11 \mathrm{a}\end{array}$ & 0 & $13 a$ & 14 \\
\hline Sustancia Vehículo 2 & $\begin{array}{c}5.03 \pm \\
0.25\end{array}$ & 1 & $12 a$ & $16 a$ \\
\hline Control Positivo (CF) ${ }^{1}$ & $\begin{array}{l}4.01 \pm \\
0.09 * a\end{array}$ & $23 * *$ & $63 * * a$ & $271 * *_{a}$ \\
\hline
\end{tabular}

$\mathrm{CP}=$ Células con Poliploidíab; $\# \mathrm{C}=\#$ de Células con aberraciones $^{\mathrm{b}}$; CF (Ciclofosfamida); ${ }^{1}$ Administración por vía intraperitoneal. aX \pm D.E. de un total de 10000 células/grupo/ serie para un total de 20000 células evaluadas; $* p<0.05$; ANOVA (Comparación contra el control negativo en cada línea evaluada y sexo); $b * * p<0.01$; prueba no paramétrica $\mathrm{X}^{2}$ (Comparación contra el control negativo en cada línea evaluada y sexo); $a=p<0.05$ (Difiere al comparar entre líneas teniendo en cuenta la misma variable. grupo experimental. prueba estadística y sexo.

totales con aberraciones en los machos y 219 en las hembras.

Al evaluar la línea de ratones OF-1 se obtuvieron mayores resultados de aberraciones de forma espontánea siendo significativos al ser comparados con Balb/c y NMRI. Se obtuvo un total de células con aberraciones que osciló 
entre $13-15$ en los machos y de $12-16$ en las hembras. Teniendo en cuenta las aberraciones inducidas por la CF se obtuvo en los machos 239 células totales con aberraciones y en las hembras 253. Resultados superiores a los obtenidos en las líneas Balb/c y NMRI en ambos sexos.

Según los resultados obtenidos en este trabajo al evaluar la línea C57BL/6/cenp en ambos sexos como biomodelo, se demostró que es la línea menos eficiente de forma espontánea y más susceptible al mutágeno. Se obtuvo de forma espontánea un total de células con aberraciones que se encuentran entre 16-17 en los machos y de $14-18$ en las hembras. La CF indujo en este biomodelo experimental una total de 254 células con aberraciones en los machos y 271 en las hembras, resultados que se encuentran por encima de los obtenidos en las líneas Balb/c, NMRI y OF-1.

No se observaron diferencias significativas al comparar los resultados entre sexos en la misma línea experimental. Siendo válidos tanto para los controles como para el grupo en el cual se indujo daño con la CF.

\section{DISCUSIÓN}

En este estudio se incluyeron animales tratados con dos sustancias vehículo, las cuales son utilizadas en la mayoría de las preparaciones tanto de productos naturales como químicos, además, una de ellas constituye el solvente del control positivo utilizado. Se decidió incluir estos grupos para analizar un mayor número de animales controles y por encontrarse estas sustancias reflejadas en la mayoría de las investigaciones que se realizan como controles negativos, gracias a su probada inocuidad.

En las tablas 1 y 2 se muestran los resultados correspondientes a la frecuencia espontánea e inducida de aberraciones cromosómicas en las cuatro líneas de ratones evaluadas. Bajo las condiciones experimentales la línea de ratones Balb/c es más eficiente para este trabajo que las demás evaluadas. Esta línea experimentó los resultados más bajos de células totales con aberraciones, que difieren con las demás líneas en ambos sexos. Estos resultados están en conjunción con los encontrados por otros autores al evaluar esta línea de ratones en biomodelos experimentales de antigenotoxicidad, donde se evalúan mutágenos reconocidos con la CF y la bleomicina $(9,10)$. Estos resultados se encentran en el rango descrito para esta especie con una frecuencia espontánea de 0-3\% células con aberraciones en 1000 células registrables (9).

Al ser comparada entre líneas de ratones la genotoxicidad espontánea e inducida teniendo en cuenta las variables numéricas I.M y el número de células con poliploidía, nuevamente la línea Balb/c experimentó los valores más altos de índice mitótico y los más bajos en el otro índice. Con este resultado se demuestran las altas tasas de crecimiento mitótico de células somáticas con bajas tasas de mutaciones numéricas. Obtener biomodelos experimentales con estos bajos índices espontáneos, permite detectar de forma más rápida y eficiente la ocurrencia de inducción de aberraciones de un producto a evaluar, lo cual hace a su vez aún más sensible y valida esta prueba.

En el caso de los resultados inducidos con CF la línea Balb/c en ambos sexos demostró ser susceptible al mutágeno evaluado, al diferir con los resultados espontáneos obtenidos en el grupo control negativo y sustancia vehículo 1 y 2. Pero la línea que demostró ser más susceptible a este clastógeno químico fue la OF-1, donde se obtuvieron los resultados más bajos de I.M como variable del daño citotóxico, lo cual manifiesta que la CF es capaz de inhibir la progresión del ciclo celular $(8,10,11)$.

Igualmente al comparar las aberraciones de tipo Gaps entre líneas de ratones tanto de espontánea como inducida, la línea Balb/c experimentó los valores más bajos con diferencias estadísticamente significativas marcadas con las demás líneas evaluadas.

Las diferencias significativas entre líneas de ratones a la acción genotóxica de la CF pudieran ser en respuesta a diferentes niveles de expresión de los genes que codifican para la enzima citocromo P-4501A1 en el hígado. Entre líneas de una misma especie se han observado diferencias epigenéticas al tener en cuenta esta enzima hepática fundamental en la fase I del metabolismo de xenobióticos, que participa en el metabolismo de la CF en el hígado al utilizarse como droga citotástica $(12,13)$.

En estudios recientes en donde se tuvo como objetivo, comparar la eficiencia del biomodelo ratas Sprague Dawley (SD) y ratones Balb/c de ambos sexos en el ensayo de micronúcleos, aberraciones cromosómicas y morfología de la cabeza del espermatozoide, nuevamente los ratones de esta línea experimentaron los índices endógenos de daño más bajos e inducidos altos $(14,15)$. 
Subrayando una vez más nuestra teoría de que genéticamente la línea de ratones Balb/c en ambos sexos es más estable que las otras evaluadas y que las ratas SD, además de haber presentado una respuesta aceptable a la acción de la sustancia mutagénica utilizada.

Además en un estudio comparativo entre estas mismas líneas teniendo en cuenta el ensayo cometa alcalino de leucocitos de sangre periférica, el ensayo de micronúcleos en células de la médula ósea y el ensayo de la morfología de la cabeza del espermatozoide arrojaron nuevamente que la línea Balb/c es la más apropiada a utilizar en los ensayos de genotoxicidad al utilizar ratones como biomodelos (16-18).

El no haber encontrado diferencias entre sexos en los grupos experimentales utilizados para cada línea de ratones, indica que no existe sexo dependencia. La respuesta endógena e inducida es dependiente del sexo. En ensayos similares pero evaluando la acción genotóxica de fármacos mediante está técnica tampoco fueron observadas diferencias entre sexos tanto en los grupos controles, tratados y grupo control positivo administrado con CF por igual vía $(8,11,14,19,20)$.

El hecho de que los ratones Balb/c hayan resultado ser la línea más apropiada en este ensayo que las otras líneas de ratones y ratas SD al tener en cuenta los índices endógenos evaluados, pudiera estar dado por la baja tasa de variabilidad genética de esta línea al ser isogénica. Esto constituye una ventaja al evaluar el efecto genotóxico de productos farmacéuticos en proceso de investigación mediante este ensayo, pero a su vez no se obtendrá una respuesta heterogénea, la cual es típica en poblaciones humanas.
Mediante este análisis se sugiere que la línea de ratones Balb/c, es la apropiada para utilizar como biomodelo en el ensayo de aberraciones cromosómicas, cuando es utilizada la CF como control positivo por la vía y dosis ensayadas. Siendo útiles estos hallazgos al evaluar mutágenos con similar mecanismo de daño al ADN. Cabe destacar que la $\mathrm{CF}$ es un agente alquilante que forma monoaductos y enlaces cruzados entre cadenas como consecuencia de la aparición de rupturas (efecto de los mecanismos de reparación) $(16,17)$. La CF pertenece al grupo de cloroetilaminas. Con el desarrollo de este agente se logró mayor selectividad de la droga hacia el tejido tumoral. Considerado un agente alquilante bifuncional, no posee especificidad por fase alguna del ciclo celular $(16,17)$.

Estos resultados conducen a una mejora en la robustez de esta prueba, ya que permiten detectar en un estrecho margen de error aquellas sustancias que sean clasificadas de muy baja genotoxicidad, además de responder de forma aceptable a clastógenos químicos como la CF (21). Además se recomienda que para un estudio más completo se pudiera evaluar la respuesta inducida con el uso de otros mutágenos reconocidos en ensayos de genotoxicidad in vivo.

En conclusión estos resultados demuestran que genéticamente la línea de ratones Balb/c en ambos sexos es más estable que las otras evaluadas, siendo el biomodelo ideal para el ensayo de aberraciones cromosómicas en células de la medula ósea cuando se realice con ratones como biomodelos. Este resultado permitirá detectar en un estrecho margen de error aquellas sustancias que sean clasificadas de muy baja genotoxicidad, puesto que se logra mayor sensibilidad y robustez en la técnica citogenética realizada.

\section{REFERENCIAS}

1. Arencibia DF, Rosario LA, Morffi J, Curveco D. Estrategias en las evaluaciones genotóxicas. Retel 2009; 23(3):23-40.

2. OECD. Guideline for the testing of chemical. Directrices de OECD TG 475, Genetic Toxicology: In vivo Mammalian Chromosome Aberration Test, in bone marrow cells. Anexo B11; USA; 1997.
3. Arencibia DF, Rosario LA, Vidal A. The mouse as biomodel in genotoxicity assays, two years of experience, Finlay Institute, Cuba. VacciMonitor 2010; 19(Supl 2):245.

4. CCAC. Canadian Council on Animal Care. Guidelines for the use of animals in Psychology. In: Olfert ED, Cross BM, McWilliam DVM, McWilliam AA (Eds.) Canada, Ottawa: Bradda Printing Services Inc: 1997. 
5. Arruzazabala ML, Mas R, Molina V. Effect of D-004, a lipid extracts from the Cuban royal palm fruit on atypical prostate hyperplasia induced by phenylephrine in rats. Drugs R\&D 2006; 7:233-241.

6. Shayne CG. Animal Models in toxicology. Chapter 2: The Mouse. Toxicology. Second edition. New York (U.S.A): Published by Shayne C. Gad and Taylor \& Francis Group, LLC; 2007.

7. Arencibia DF, Rosario LA, Morffi J, Curveco D. Desarrollo y estandarización de la técnica en tres ensayos de genotoxicidad. Retel 2009; 25(3):22-38.

8. Arencibia DF, Gámez R, Gutiérrez $A$, Mas $R$, Pardo B, García H, et al. Efectos del D-003, mezcla de Ácidos Alifáticos en el ensayo de Aberraciones Cromosómicas in vivo. Rev Cub Farm 2010; 44(2):213-220.

9. Kramer PJ. Genetic toxicology. The in vitro and in vivo aberration test. J Pharm Pharmacol 2000; 4:395-405.

10. Arencibia DF, Rosario LA, Curveco D. Comparación de la respuesta de ratones Balb/c de ambos sexos a la administración de dos sustancias mutagénicas mediante el ensayo de aberraciones cromosómicas en células de la médula ósea. Rev Vet Arg 2010; 27(269):1-10.

11. Gutiérrez A, Gámez R, Arencibia DF, Pardo B, García H. Evaluación del potencial genotóxico del D-004, para inducir aberraciones cromosómicas en médula ósea de ratones. Rev CENIC 2010; 41(Especial):1-7.

12. Amri $H$, Batt A, Siest G. Comparison of cytochrome $\mathrm{P}-450$ content and activities in liver microsomes of seven species including man. Xenobiotica 1986; 16:351-358.

13. Jana NR, Sarkar S, Yonemoto J, Tohyama C, Sone H. Strain differences in cytochrome P451A1 gene expression caused by 2, $3,7,8$-tetrachlorordibenzo-p-dioxin in the rat liver: Role of the aryl hydrocarbon receptor and its nuclear translocator. Biochem Biophys Res Comun 1998; 248:554-558.
14. Arencibia DF, Rosario LA, Suárez YE, Vidal A. Comparación entre dos biomodelos murinos en el ensayo de micronúcleos y de aberraciones cromosómicas en células de la médula ósea. Química Viva 2011; 10(2):106-117.

15. Arencibia DF, Vidal A, Rosario LA, Suárez YE, Delgado L. Biomodelos para la inducción de micronúcleos en células de la médula ósea por ciclofosfamida y bleomicina. VacciMonitor 2011; 20(1):28-33.

16. Arencibia DF, Rosario LA, Rodríguez Y. Assessment of basal and induced DNA damage on lymphocytes from three mouse lines by means of the alkaline comet assay. Biotecnología Aplicada 2011; 28(2):101-105.

17. Arencibia DF, Rosario LA, Suárez YE. Sensibilidad de distintas líneas murinas a la ciclofosfamida medida a través del ensayo de micronúcleos en células de la médula ósea. Journal of Basic \& Applied Genetics 2011; 22(2):125-132.

18. Arencibia DF, Rosario LA, Suárez YE. Comparación entre líneas de ratones como biomodelos en el ensayo de la morfología de la cabeza del espermatozoide. Rev Int Androl 2011; 9(3):103-108.

19. Gocke E, Bürgin H, Müller L, Pfister Th. Literature review on the genotoxicity, reproductive toxicity, and carcinogenicity of ethyl methanesulfonate. Toxicol Lett 2009; 190:254-265.

20. Sandeep D, Krishnan CH. Radioprotection by $\alpha$-asarone: Prevention of genotoxicity and hematopoietic injury in mammalian organism. Mutat Res 2011; 722:62-68.

21. Rezvanfar MA, Sadrkhanlou RA, Ahmadi A, Shojaei H, Rezvanfar MC, Mohammadirad A. Protection of cyclophosphamide-induced toxicity in reproductive tract histology, sperm characteristics, and DNA damage by an herbal source; evidence for role of free-radical toxic stress. Human Exp Toxic 2008; 27:901-910. 